\title{
Showing cytokines the way out
}

Activation of T helper $\left(\mathrm{T}_{\mathrm{H}}\right)$ cells results in the secretion of various cytokines that can determine the fate of an immune response. These cytokines are secreted into areas that are tightly packed with other immune cells, so how do T cells ensure target-cell specificity? Reporting in Nature Immunology, Huse et al. have identified a mechanism by which the specificity of intercellular communication is maintained. They describe two directionally distinct cytokine secretion pathways in $\mathrm{T}_{\mathrm{H}}$ cells: one towards the immunological synapse - a distinct region formed at the contact zone of the T cell and antigen-presenting cell resulting from the specific reorganization of cell-surface proteins - and another multidirectional pathway.

In this study, Huse et al. show, using intracellular cytokine staining, that the secretion of interleukin-2 (IL-2) and interferon- $\gamma($ IFN $\gamma)$, as well as IL-3 and IL-10, is focused at the immunological synapse in activated $\mathrm{T}_{\mathrm{H}}$ cells. By contrast, tumour-necrosis factor (TNF) was shown to be focused at the immunological synapse briefly, but as the response developed, TNF became scattered throughout the cell. Using video microscopy, TNF secretion by live $\mathrm{T}_{\mathrm{H}}$ cells was shown to be distributed throughout the cells, with a bias away from the immunological synapse over time. The intracellular distribution of CC-chemokine ligand 3 (CCL3) and CCL5 was similar to TNF, although with delayed kinetics. In addition, the $\mathrm{T}_{\mathrm{H}} 2$-cellassociated cytokine IL-4 was found to be distributed throughout the cell.

But how does the cell coordinate the movement of certain cytokines in one direction while sending others on a different route? This study shows that specific trafficking proteins co-localize with certain cytokines at these different locations. RAB3D and RAB19, members of the RAB family of small GTPases
— which associate with different intracellular compartments - were found to localize with IL-2 and IFN $\gamma$ at the synapse, whereas the SNARE (soluble $N$-ethylmaleimide-sensitive accessory-protein receptor) protein, syntaxin 6 , localized with TNF throughout the cell, indicating the use of distinct processes for directional cytokine release.

Together, these results show that T cells differentially direct cytokines, using specific trafficking proteins, through two distinct release pathways. One pathway focuses certain cytokines to the immunological synapse, where localized cell-cell communication can occur, whereas the other pathway releases cytokines, that are mainly associated with inflammation and cell recruitment, into the surrounding milieu in a multidirectional fashion. Therefore, the $\mathrm{T}_{\mathrm{H}}$-cell-cytokine response might not depend solely on the type of cytokines induced but also on the method of their release.

Olive Leavy

ORIGINAL RESEARCH PAPER Huse, M., Lillemeier, B. F., Kuhns, M. S., Chen, D. S. \& Davis, M. M. T cells use two directionally distinct pathways for cytokine secretion. Nature Immunol. 29 Jan 2006 (doi:10.1038/ni1304)

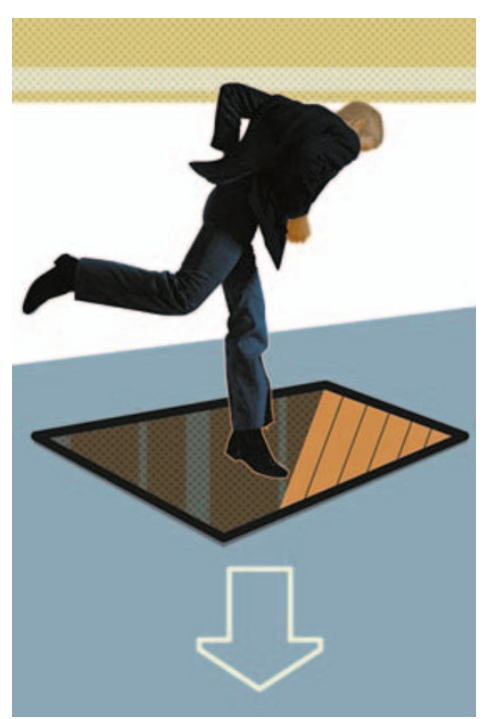

IN BRIEF

$\Rightarrow$ DEVELOPMENT

Langerhans cells arise from monocytes in vivo.

Ginhoux, F. et al. Nature Immunol. 29 Jan 2006 (doi:10.1038/ni1307)

Despite numerous attempts, the circulating precursors that give rise to skin-resident dendritic cells, known as Langerhans cells (LCs), have not been unequivocally defined. Using fluorescent latex beads, the authors tracked the fate of individual precursor populations in mice subjected to skin injury, which eliminates LCs and induces repopulation by circulating precursors. Monocytes that express high levels of Gr1 homed to inflamed skin and proliferated in situ before differentiating into LCs in 2-3 weeks, indicating that $\mathrm{Gr} 1^{\text {hi }}$ monocytes are direct precursors of LCs. The receptor for colony-stimulating factor 1 (CSF1R) was shown to be crucial for this process, as CSF1R-deficient bone-marrow progenitors failed to reconstitute the LC pool after skin injury.

\section{AUTOIMMUNITY}

Blocking the $\alpha 4$ integrin-paxillin interaction selectively impairs mononuclear leukocyte recruitment to an inflammatory site.

Féral, C. C. et al. J. Clin. Invest. 9 Feb 2006 (doi:10.1172/JCl26091)

Reagents that block the interaction between $\alpha_{4}$-integrin and VCAM1 (vascular cell-adhesion molecule 1) have been used to treat human autoimmune diseases, such as multiple sclerosis, because of their ability to inhibit the recruitment of lymphocytes to sites of inflammation. However, this therapy can cause adverse side effects, such as impaired immunity and haematopoiesis. To avoid this, the authors generated mice with a mutation in $\alpha_{4}$-integrin that blocks its ability to bind the signalling adaptor protein paxillin. This specifically inhibited the migration of lymphocytes and monocytes to the peritoneum in response to thioglycollate-induced inflammation, but it did not affect their homing to lymph nodes, the generation of humoral immune responses or haematopoiesis, which were all normal in the $\alpha_{4}$-integrinmutant mice. So, pharmaceutical targeting of the $\alpha_{4}$-integrin-paxillin interaction might be important for future treatments of inflammatory disease.

\section{CELLS}

Complement-induced regulatory T cells suppress T-cell responses but allow for dendritic-cell maturation.

\section{Barchet, W. et al. Blood 107, 1497-1504 (2006)}

Activation of T cells through the T-cell receptor and CD46a complement regulator - induces T cells with a regulatory phenotype that produce high levels of interleukin-10 (IL-10) and suppress the activation of bystander T cells. Barchet et al. examined the effect of these CD46-induced regulatory-like T cells on dendritic cells (DCs) and found that the maturation of DCs was not suppressed. In addition to producing high levels of IL-10, these T cells secrete granulocyte/macrophage colony-stimulating factor (GM-CSF) and soluble CD40 ligand, which were shown to function as a growth factor and to reverse the inhibitory effects of IL-10 on DCs, respectively. Therefore, through a distinct cytokine profile, these complement-induced regulatory-like T cells allow DC maturation but inhibit T-cell responses. 